\title{
Bases de datos para la historia cultural. Crítica literaria y periodismo en Destino
}

\author{
Databases for Cultural History. Literary Criticism and Journalism \\ in Destino
}

\author{
Blanca Ripoll Sintes \\ Universitat de Barcelona, España
}

\begin{abstract}
Resumen: Los Estudios Culturales a partir de la prensa periódica ofrecen al investigador una multitud de datos que, en ocasiones, puede generar confusión o errores, si no se clasifican y ordenan de forma rigurosa. Este trabajo pretende exponer la creación de una base de datos para el estudio de la crítica literaria en el semanario Destino, como ejemplo de utilización de esta herramienta en la Historia Cultural.

Palabras clave: estudios culturales, periodismo, bases de datos, Destino, franquismo.
\end{abstract}

Abstract: Cultural Studies done through journals and media provide the researcher with large amount of data which can occasionally create confusion or mistakes, if they are not classified and ordered in a rigorous way. This work aims to present our database, born to study Literary Criticism through the weekly magazine Destino, as an example of database's application in Cultural History.

Keywords: Cultural Studies, Journalism, Database, Destino, Franquism.

\section{Estado de la cuestión. Origen y problemática de la base de datos de Destino}

Cuando nos enfrentamos ante el posible estudio de la crítica literaria realizada en una revista semanal publicada durante casi cuarenta años (revista no digitalizada entonces), surgió como un imperativo categórico la necesidad de organizar en una herramienta informática una cantidad ingente de datos que no habían sido sistematizados hasta entonces. Contábamos con estudios globales acerca de la historia de la publicación (Geli \& Huertas Clavería, 1991; Porcel, 2003), sobre la historia política del semanario (Thomàs, 1992), U otras aproximaciones parciales (Cabellos i Minguez \& Pérez i Vallverdú, 1987: 19-36; Guillamet, 1996; Cabo, 2001). Sin embargo, ninguno de estos estudios brindaba tablas o listas de referencias en los que estuvieran consignadas todas las entradas periodísticas que conformaron la intrahistoria de Destino.

Sin lugar a dudas, no podía ser tarea de un solo individuo crear un listado que incluyera todas las secciones del semanario barcelonés. Podría haber sido objeto para un grupo de investigación que, además, contara con un técnico 
informático -quede esta brecha para futuros investigadores-. Por este motivo, nos propusimos estudiar la crítica literaria en Destino y escogimos la herramienta de la base de datos informática para organizar los datos que aparecerían del vaciado.

Esta labor se enmarca en el proceso de investigación doctoral que dio como resultado nuestra tesis La crítica de la literatura española en el semanario "Destino" (1939-1968). La novela (Ripoll Sintes, 2011). El artículo que presentamos parte de dicha investigación y quiere constituirse en una descripción y una reflexión acerca de las diversas problemáticas que nacieron durante la configuración de la base de datos, así como de su funcionalidad.

Con toda certeza, la efectividad de dicha herramienta informática se confirmó durante el estudio de los datos (aumento de la agilidad en las búsquedas, del rigor en la descripción de la información) y juzgamos que fue una aportación destacable para la comunidad científica, pues, pese a que la Biblioteca Nacional de Catalunya digitalizó la revista, el buscador textual dista mucho de ser útil y fiable. En consecuencia, demostraremos cómo la digitalización de prensa periódica debe ir acompañada de un detallado buscador textual o de una minuciosa base de datos.

\section{Periodismo y crítica literaria. Los peligros de la Biblioteca de Babel}

Esta aportación al análisis de las posibilidades de investigación que la herramienta de la base de datos ofrece al historiador cultural o literario, parte de una hipótesis de trabajo fundamentada en la convicción de que la revista barcelonesa Destino no solo fue un mirador privilegiado desde donde se puede apreciar la realidad española de la época desde ópticas tan diversas como la política nacional e internacional, la sociedad, el pensamiento, el mundo del espectáculo o de la cultura. Destino fue, además, un agente protagonista en la configuración de la realidad cultural de su tiempo: contribuyó de forma notable a la creación del canon narrativo de la España de la posguerra que ha llegado hasta nuestros días y albergó entre sus páginas a otros nombres propios, olvidados por el canon actual, pero que fueron relevantes en su época y merecen, quizá, un lugar más destacado en el panorama literario.

El estudio realizado se sirvió de la perspectiva de la crítica literaria ejercida desde el semanario, para analizar la creación narrativa durante las tres primeras décadas de la dictadura franquista. No obstante fue fundamental atender otras secciones de la revista, más allá de las meramente literarias: los editoriales, columnas de escritores, colaboraciones literarias, entrevistas, crónicas, etc. En este sentido, las intersecciones que la óptica de la Historia Cultural nos ofrece hallan su máxima representación y sus mayores complejidades en el estudio de la prensa periódica: la publicidad editorial, la sociología literaria, la crítica o la configuración de un estado de opinión en lo que Habermas (1994) denominó la "esfera de lo público", se entretejen en un bosque en el que es fácil perderse.

La necesidad de reseguir con rigor y detalle el devenir cotidiano de la recepción crítica en la revista nos obligó a contar con herramientas que ordenaran, secuenciaran y clasificaran los datos que 30 años de una revista semanal nos proporcionaba: del primer número publicado en Barcelona, el 
101 (después de 100 números del semanario creados en Burgos durante la Guerra Civil, como Boletín de la F.E.T. y de las J.O.N.S.) hasta el 1630 contamos con 1529 volúmenes de semanario que generarían una base de datos de más de 5.000 entradas.

Esta cantidad de información nos proporcionó un caleidoscopio de nombres propios y títulos de novelas que, si por una parte contaba con ventajas como la inmediatez de la recepción semanal y de la importancia cuantitativa, también conllevaba ciertos peligros: el ingente número de artículos, crónicas, columnas y reseñas podía provocar que nuestra tesis se convirtiera en un gran registro, en una gran enumeración de referencias, en el que estuviera ausente la abstracción necesaria para dar trascendencia y vigencia al estudio.

Para salvar este escollo, se añadió, a la ordenación cronológica, una estructuración temática, conscientes del anacronismo y de la naturaleza ficcional de dichos marbetes estéticos, dados, en muchas ocasiones, a posteriori del hecho literario. Quizá quepa reflexionar, llegados a este punto, en torno a la naturaleza misma de la historia cultural o de la historia literaria. En este sentido, el profesor Gies lanzaba estos lúcidos interrogantes al historiador moderno en su artículo "El Efecto Funes", jugando con la contraposición entre Pierre Menard y Funes el memorioso, protagonistas de sendas Ficciones borgeanas:

Si Menard no podía (¿̇cómo no? ) revivir cada momento, cada detalle de la vida del autor de Don Quijote, para poder imitar no sólo el producto final (el libro) sino también las experiencias que informaron dicho libro, y Funes no podía olvidar nada (y, por consiguiente, no pensar), ¿̇qué le ocurre, entonces, al historiador moderno de la literatura, que se enfrenta con retos parecidos? ¿Cómo puede el historiador de la literatura 'pensar' cuando se ve abrumado por una avalancha de detalles sin orden (fechas, categorías, nombres, títulos, 'ismos', movimientos, lenguas, escuelas, nacionalidades)? Para escribir la historia literaria -reescribir la historia literaria- ihace falta volver a vivir la historia? (...) Como Funes, ¿estamos perdidos si intentamos recordar nuestra historia literaria e igualmente perdidos si no la intentamos recordar? (Gies, 2009: 9)

Con él, debemos preguntarnos: ¿̇Debe ser la historia literaria o cultural un inmenso hipertexto en el que encontremos todos los datos existentes? Aceptando la posibilidad de que esta enorme base de datos pudiera crearse, ¿seríamos capaces de aprehender el conocimiento allí cifrado? O, quizá, en palabras de Gies:
(...) ¿lllegaremos entonces a otro momento borgeano, el de la Biblioteca de Babel, en el cual tenemos acceso a toda la información del mundo desde siempre y así al infinito, y sin embargo, en donde precisamente por ser mera acumulación de detalles (los detalles, de nuevo), no tenemos acceso a nada? (Gies, 2009: 16)

Lejos de los extremos de la Biblioteca de Babel, de Funes el memorioso o de Pierre Menard, autor del Quijote, nuestra tesis partió de una premisa de base, que Gies bautizaba en su artículo, muy acertadamente, como "la inquietud de la exclusión". Así pues, desde la certeza de la imposibilidad del 
conocimiento totalizador, construimos una base de datos informática que permitiera filtrar y rastrear de forma más rápida la información resultante del vaciado de la revista Destino.

\section{La gestación de la base de datos}

Nuestra investigación abarcó cuatro géneros literarios durante la fase del vaciado y clasificación de los materiales de la revista. Dichos géneros fueron: la novela, el relato breve, la poesía y la literatura de viajes. Cuando se completó el vaciado para la elaboración de la tesis doctoral, la cantidad de información superó con creces nuestras expectativas de modo que suprimimos del análisis que completaba la base de datos los géneros de la poesía y de la literatura de viajes. Con todo, sí estaban presentes en las más de 5.000 entradas que configuraron nuestro archivo informático.

Era importante señalar esta cuestión para dotar de sentido la articulación de la base de datos. Cada entrada se estructuró a modo de referencia bibliográfica ampliada con otros factores de análisis importantes para nuestro estudio:

a. Autor (del artículo o reseña);

b. Título (del artículo, reseña o colaboración);

c. Sección (de la revista);

d. Páginas;

e. Número;

f. Fecha (referencia completa: DD/MM/AAAA);

g. Género Periodístico (crítica, reseña, artículo, crónica, columna, colaboración, publicidad, nota o reportaje);

h. Género Literario (de la obra en cuestión analizada o referenciada en el texto de la entrada; en nuestro caso, barajábamos cuatro géneros: novela, relato breve, poesía y literatura de viajes);

i. Palabra Clave (concepto o nombre propio que resuma la materia del artículo);

j. Materia (disciplina académica a que se hace referencia en el texto);

k. Director de la revista (Ignacio Agustí y Néstor Luján serán los dos grandes directores de Destino; Álvaro Ruibal se hizo cargo del largo paréntesis que Agustí pasó en Suiza: entre el número 272 - 03/10/1942 y el número 345 26/02/1944);

I. Observaciones (en esta columna, se aportó información necesaria cuando el título del artículo era excesivamente neutro; no implicaba que en todas las entradas existiera un resumen del artículo en cuestión, para evitar que la base de datos se convirtiera en un archivo inmanejable);

m.Clasificación (para agilizar ciertas búsquedas, se optó por tres posibilidades: "Crítica literaria", objeto principal de nuestro estudio; "Crónica cultural", artículos, columnas, editoriales o noticias de carácter general; y "Vida 
literaria", marbete que se refiere a todo el resto de crónicas, ecos, entrevistas, noticias o artículos relacionados con el mundo de la literatura).

Aportamos un ejemplo gráfico (dividido en dos partes) de una entrada de la base de datos:

\begin{tabular}{|c|c|c|c|c|c|c|}
\hline Autor & Título & Sección & Páginas & Número & Fecha & $\begin{array}{c}\text { Género } \\
\text { Periodístico }\end{array}$ \\
\hline Redacción & \begin{tabular}{|ll} 
Gabriel & Avilés \\
"Tribunales rojos vistos \\
por un abogado \\
defensor. Un reportaje \\
de héroes y mártires..."
\end{tabular} & & 3 & 101 & $24 / 06 / 1939$ & Publicidad \\
\hline
\end{tabular}

\begin{tabular}{|c|c|c|c|c|c|}
\hline \begin{tabular}{|l|} 
Género \\
Literario
\end{tabular} & $\begin{array}{l}\text { Palabra } \\
\text { Clave }\end{array}$ & Materia & Director & Observaciones & Clasificación \\
\hline Novela & $\begin{array}{l}\text { Gabriel } \\
\text { Avilés }\end{array}$ & $\begin{array}{l}\text { Literatura } \\
\text { Española }\end{array}$ & \begin{tabular}{|l|} 
Ignacio \\
Agustí
\end{tabular} & $\begin{array}{l}\text { Novela-reportaje. Recibió } 3 \text { críticas } \\
\text { literarias en Levanter (Valencia), } \\
\text { Solidaridad Nacional (Barcelona) y } \\
\text { La Vanguardiar Española } \\
\text { (Barcelona) }\end{array}$ & Vida literaria \\
\hline
\end{tabular}

Figura 1. Primera entrada de la base de datos de "La crítica de la literatura española en el semanario "Destino" (1939-1968). La novela" (2011).

Como puede apreciarse con un solo ejemplo, la elaboración de cada una de las entradas implicaba la localización, lectura y análisis previo de cada uno de los textos (para el estudio que precedía, en la tesis, a la base de datos digital, en CD adjunto, se procedió a la transcripción y análisis de todos los artículos vaciados).

Se eligió como herramienta informática la base de datos relacional Access del paquete ofimático de Microsoft Word, que era el que más se adaptaba a las condiciones formativas (puesto que desconocemos por completo los vericuetos del mundo de la programación) y materiales de nuestra investigación predoctoral. El modelo Access nos permitía establecer relaciones entre la información, que estaba dispuesta en tablas, y así poder llegar a cruzar distintas tablas a partir del mismo instrumento.

A medida que se iban engrosando las filas del archivo, aparecían los primeros conflictos referentes a cuestiones de clasificación o designación. Para que una base de datos informática sea realmente efectiva, es condición sine qua non que se rija por el principio de la coherencia y la homogeneidad de criterios.

En este sentido, la primera problemática que surgió fue la que resultaba de las intersecciones presentes entre los campos culturales catalán y español. Destino fue una revista fundada por catalanes huidos de la Barcelona republicana y reunidos en el Burgos sublevado en torno a la Delegación de Prensa y Propaganda de la Territorial catalana de Falange, capitaneada por el poeta Dionisio Ridruejo. Así, catalanes y españoles, añorados de su tierra y reivindicadores de su españolidad, el idioma español se impuso como vehículo de expresión y comunicación, y así prosiguió, como no podía ser de otro modo 
dentro de la ortodoxia franquista, durante toda su trayectoria hasta que desaparece en 1975, tras la venta de acciones por parte de su gerente Josep Vergés. Sin embargo, la mayoría de redactores y colaboradores eran individuos formados en la Barcelona de los años veinte y treinta, en numerosas publicaciones escritas en lengua catalana y habían utilizado dicho idioma en su faceta como escritores y periodistas; muchos de ellos recuperarían su idioma materno cuando, después de 1946, se flexibilizó mínimamente el uso del catalán en el ámbito de la creación literaria.

¿Cómo debíamos introducir los nombres propios de estos escritores, a medio camino de ambos idiomas? Durante las dos primeras décadas, la mayoría de nombres propios se españolizan en Destino. Así, Ignasi Agustí firmaba como Ignacio; Josep Pla como José; o Joan Teixidor, como Juan. Pero con el paso de los años, algunos de ellos pasaron a firmar sus textos con el nombre propio en catalán. Si queríamos, en un momento determinado, filtrar la base de datos desde la columna de un autor determinado, para así obtener todos los textos firmados por dicho personaje, debíamos uniformizar el criterio de denominación. Se optó por los nombres propios catalanes, pues tuvimos en cuenta que la españolización onomástica se debía a las circunstancias históricas del Franquismo.

A esta cuestión, debíamos sumar otro hecho propio de la época histórica: la proliferación de pseudónimos. Cualquier atisbo de sospecha o dato confirmado que situara a un periodista cerca de las instituciones de la II República Española o de cualquier organización sindical u obrerista, era suficiente para que se le prohibiera el ejercicio del periodismo. Era tan simple como la denegación del carné obligatorio para colaborar en cualquier medio de comunicación. En consecuencia, numerosos intelectuales y periodistas se vieron ante la necesidad de firmar con un alias para poder sobrevivir. En los casos en que siempre la máscara utilizada fuera siempre la misma, no existía disparidad de denominación. Es lo que ocurre, por quedarnos con un ejemplo, con Manuel Brunet, que firma todos los textos de la revista como "Romano". En la base de datos, en el apartado de Autor, aparecía toda la información necesaria para la identificación (Brunet, Manuel "Romano"), que siempre debíamos escribir, con pulcritud y rigor, de la misma forma, para que, al filtrar las entradas, no se generaran errores.

Sin embargo, ¿̇cómo proceder en las situaciones en que una misma persona escribiera con diferentes pseudónimos? ¿ $\bigcirc$ cuando un periodista del núcleo de Destino se desdoblaba bajo otros alias para generar el efecto de variedad en la menguada redacción del semanario? Nuestro objetivo seguía siendo que, mediante la herramienta del filtro de la base de datos, pudiéramos obtener listados a partir de criterios definidos de búsqueda. La homogeneidad seguía siendo esencial. En aras de lograr dicho propósito, se decidió que, como Autor, apareciera siempre el nombre propio real a pesar de que no hubiera firmado el texto. En el apartado de Observaciones, se consignaría el pseudónimo y se daría información de todas las máscaras con las que contaba cada autor en el vaciado de la revista. Así, nos encontramos con casos como el de Antonio Espina, que firmó en el semanario barcelonés con pseudónimos como "Aristeo" o, el más frecuente, "B. Ruiz Soto", ante la prohibición de hacerlo con su nombre real por su significación republicana 
antes de la contienda. Y, por dar una muestra de la segunda posibilidad, hallamos la variedad de nombres bajo los que se multiplicó Ignasi Agustí: "Gerardo Juan", "Gustavo Riff" o "GIN". Para lograr una identificación fidedigna de los pseudónimos presentes en la revista Destino fueron insoslayables los estudios precedentes de Carles Geli y José María Huertas Clavería, Las tres vidas de Destino (1991: 26-27), y de Joan Maria Thomàs, Falange, guerra civil, franquisme (1992: 300 y ss.).

En ocasiones, la situación se complica con la utilización de siglas, bien del nombre y apellido, bien solo del apellido. Para la configuración del archivo digital se procedió del mismo modo que con los pseudónimos. Por último y en lo referente a la designación autorial, un hecho recurrente en los estudios periodísticos y que aumenta a medida que retrocedemos en nuestra historia es la notable cantidad de textos sin firmar. Durante la época de la posguerra, este acontecimiento se debía, principalmente, a dos motivos: bien a que el texto había surgido de algún miembro de la redacción de la revista (que solía corresponderse con algún joven ayudante), bien porque se trataba de la reelaboración de una nota informativa comprada a diversas agencias de noticias. Para solventar esta habitual situación, escogimos el concepto "Redacción" para englobar esta casuística.

Conflictos similares surgieron en otras variables de clasificación, como el Género Periodístico. La velocidad connatural de los medios de comunicación, su flexibilidad y porosidad genéricas, así como la historia demasiado reciente de fórmulas modernas como el reportaje, son algunos de los factores que problematizaron la clasificación uniforme de ciertos textos que podían ir a caballo de dos posibilidades. Con todo, se justificó, a partir del apartado de Observaciones, cada elección.

Como puede deducirse de lo desgranado hasta aquí, la base de datos se creó partiendo de unos criterios de ordenación determinados, pero fue modificándose a medida que se avanzaba en el cotejo y vaciado de la revista, conformándose casi como un organismo vivo que debía adaptarse a cada nueva circunstancia y revisarse, desde la primera entrada hasta la última, en función de cada nueva decisión que como investigadores nos veíamos obligados a tomar.

La funcionalidad de la base de datos creada se vio confirmada muy pronto y consolidada en posteriores investigaciones -doctorales y postdoctorales-. No solo establecía parámetros rigurosos de localización de los textos, sino que brindaba una gran cantidad de información de forma sintética y gráfica. Asimismo, permitió agilizar búsquedas muy específicas (a partir de los diversos parámetros establecidos: por autor, por género literario, etc.), con lo que podíamos filtrar información muy concreta en un bosque de referencias en el que era fácil perderse.

\section{Una inversión para el futuro: posibilidades de las bases de datos en la historiografía cultural}

Son estos malos tiempos, ya no para la lírica, sino para los estudios culturales a partir de prensa periódica. En nuestra labor como docentes y directores de trabajos finales de Grado o Máster no es frecuente hallar en los estudiantes la disposición y la constancia necesarios para enfrentarse a la tarea a veces 
severa de rastrear hemerotecas, abrir periódicos y revistas, y proceder a un estudio exhaustivo.

Sin embargo, las posibilidades que este ámbito ofrece son de largo recorrido. En primer lugar, porque los medios de comunicación escritos nos permiten asistir a la gestación en vivo, en caliente, del fenómeno literario o cultural: quién dijo qué, en qué momento, por qué razón. Un estudio palpitante de la realidad cultural de un tiempo que a veces resitúa, corrige y siempre amplía y confiere profundidad a ciertos estudios de historia literaria o cultural que, por falta de tiempo, distancia o rigor, asumen ciertos presupuestos sin cotejarlos con las fuentes originales. En segundo lugar, la prensa periódica nos ofrece una perspectiva poliédrica que tan idónea resulta para los estudios culturales; puesto que la profesionalización de los escritores se da la mano, en las páginas de una revista, con las leyes socioeconómicas que dictan la publicidad; la crítica literaria se abraza a la crónica teatral o cinematográfica; la crítica musical, con la crónica artística; la opinión política con temas de sociedad; etc.

Acto seguido, no es baladí recordar que un estudio pormenorizado de una publicación (a no ser que su trayectoria sea breve) nos ofrece una interesante cantidad de materiales, muchos de ellos seguramente inéditos y otros, carentes de contexto, que podrán nutrir nuestra labor investigadora en el futuro. De esta forma, la rentabilidad posterior de la labor de hemeroteca es directamente proporcional a las horas invertidas en un comienzo.

Y, a pesar de todos estos motivos, nada de ello podría hacerse de forma rigurosa y rápida sin contar con herramientas informáticas a nuestro alcance, como las bases de datos. Dicho instrumento permite, tras una correcta, detallada y coherente introducción de la información recolectada, búsquedas ágiles que ahorrarán tiempo, esfuerzo y olvidos al investigador. Cuanto más completa sea la base de datos, cuantas más tablas incluya, más posibilidades relacionales ofrece. Asimismo, el investigador obtendrá un mayor rendimiento si ha dedicado tiempo a priori para incluir todos los parámetros de análisis posibles. Por ejemplo, en nuestro cotejo podíamos filtrar a partir de la columna Autor, pero dentro de la columna Género Literario podíamos separar cuántas entradas (o filas) pertenecían al género de la poesía (o del relato, o de la literatura de viajes...); podíamos detectar, en una sola pantalla, las referencias a un único autor literario, o a una obra determinada; o podíamos seleccionar por Género Periodístico cuántas entrevistas se habían publicado.

Con todo, somos conscientes de que otro tipo de programa informático (FileMaker, bases de datos orientadas a grafos, etc.) nos permitiría una presentación gráfica mucho más atractiva y quizá vinculable a presentaciones en páginas web de acceso público. En el caso concreto de nuestra investigación, era interesante construir una base de datos relacional que agilizara la localización de información en una notable cantidad desorganizada de datos, pero no tanto una representación gráfica de dicha información. En este sentido, una opción que necesariamente debería englobar a un colectivo de trabajadores sería poder dotar de profundidad y conexiones a nuestra tabla de datos con enlaces que los vincularan a la digitalización de la revista Destino, presente en ARCA (http://mdc2.cbuc.cat/cdm/search/collection/destino), de manera que 
nuestra labor pudiera ser de mayor utilidad para toda la comunidad académica y alcanzara una mayor visibilidad y diseminación.

Recuperemos, para finalizar, las reflexiones que David Gies lanzaba en "El Efecto Funes". Una base de datos puede ser una auténtica Biblioteca de Babel. Es misión de su creador elaborar estrategias que faciliten el rastreo y la búsqueda de información: la homogeneidad de criterios, la rigurosidad historiográfica en el vaciado. Y, en definitiva, es función del historiador cultural o literario dotar de trascendencia a un mero registro de datos a través del análisis, la reflexión, el establecimiento de vínculos y conexiones, que consigan vivificar el texto encasillado en filas y columnas, para que cobre sentido y vigencia a ojos del lector.

\section{Bibliografía}

CABELlOS I MINGUEZ, P. \& E. PÉREZ I VALLVERDÚ (1987): "Destino. Política de unidad (1939-1946). Tres aspectes de l'inici d'una transformació obligada". Els Marges, 37, pp19-36.

CABO, I. (2001): La resistencia cultural bajo el franquismo. Barcelona: Altera.

GELI, C. \& J. M. HUERTAS CLAVERÍA (1991): Las tres vidas de Destino. Barcelona: Anagrama.

GIES, D. T. (2009): "El Efecto Funes. La Historia Literaria en el Siglo XXI". La Obra en Marcha: ensayos en homenaje a Richard Cardwell. J. ANDREWS \& S. G. H. ROBERTS (eds.). Nottingham: Critical, Cultural and Communications Press, pp. 8-19.

GUILLAMET, J. (1996): Premsa, franquisme i autonomia. Crònica catalana de mig segle llarg (1939-1995). Barcelona: Flor del Viento.

HABERMAS, J. (1994): Historia y crítica de la opinión pública. México-Barcelona: GG.

PORCEL, A. (ed.) (2003): La crónica de Destino. Barcelona: Destino.

RIPOLL SINTES, B. (2011): La crítica de la literatura española en el semanario "Destino" (1939-1968). La novela. Tesis doctoral dirigida por el dr. Adolfo Sotelo Vázquez y defendida el 02/06/2011. Barcelona: Universitat de Barcelona.

THOMAS, J. M. (1992): Falange, guerra civil, franquisme. F.E.T. y de las J.O.N.S. de Barcelona en els primers anys del règim franquista. Barcelona: Publicacions de l'Abadia de Montserrat. 